\title{
Food and Drug Administration - approved molecular methods for detecting human papillomavirus infection
}

\author{
Katarzyna Sitarz ${ }^{1,2}$, Slawa Szostek ${ }^{1}$ \\ ${ }^{1}$ Department of Virology, Chair of Microbiology, Jagiellonian University Medical College, Cracow, Poland \\ ${ }^{2}$ Faculty of Chemistry, Jagiellonian University, Cracow, Poland
}

\begin{abstract}
In the world, there are many tests that allow the detection of HPV infection. These tests are based on different operating principles and have different levels of sensitivity. The first test to detect HPV infection was approved by the Food and Drug Administration in 2003. Since then, the FDA has approved five more commercial tests for this purpose, the last one in 2018. This paper discusses the principles of molecular tests to detect HPV, which have been approved by the FDA, the main differences between them, as well as their advantages and disadvantages.
\end{abstract}

Key words: HPV detection; Hybrid Capture; Cervista; COBAS; Aptima; Onclarity

Ginekologia Polska 2019; 90, 2: 104-108

\section{INTRODUCTION}

Cervical cancer is currently the fourth most common cancer in terms of both incidence as well as mortality among women in the world [1]. According to estimated data, in 2018 in the world, there were 570,000 new cases of this cancer and 311,000 deaths. However, in 28 countries, it is the most commonly diagnosed cancer among women, and in 42 countries, it is the cancer with the highest mortality rate among women [1]. In Poland, cervical cancer now ranks seventh in terms of cancer incidence and ninth as regards cancer mortality among women [2]. Main known etiological factors of cervical cancer are oncogenic types of human papillomavirus (HPV) [3]. HPV is a sexually transmitted virus. There are both high-oncogenic and low-oncogenic HPV types. The group of high-oncogenic types includes HPV $-16,-18,-31$, $-33,-35,-39,-45,-51,-52,-56,-58$ and -59 [4]. However, manufacturers of most commercial tests described here have also included the -66 and -68 types to high-risk (HR) types. In the further part of the publication, this classification will be adopted for simplification, however, these types are classified by the authors of the latest publications as probably (-66) and possibly (-68) carcinogenic [4]. The operation of molecular tests lies primarily in the detection of these types.

The important aspects in preventing the development of cervical cancer involve both cytological examination and the detection of HPV infection [5, 6]. The current guidelines of the Polish Society of Gynecologists and Obstetricians recommend performing an HPV test in case of obtaining an abnormal cytology result (ASC-US, LSIL) as an alternative to a repeat cytology test [7]. There are many methods for detecting HPV infection, which we can divide into three main groups: nucleic acid hybridization assays, signal amplification assays and nucleic acid amplification assays [5]. Until today, the Food and Drug Administration (FDA) has approved 7 tests detecting HPV infection: three signal amplification assays (Hybrid Capture ${ }^{\mathrm{TM}}$ II generation, Cervista ${ }^{\mathrm{TM}}$ HPV HR, Cervista ${ }^{\mathrm{TM}}$ HPV 16/18) and four nucleic acid amplification assays (COBAS ${ }^{\circledR}$ HPV Test, Aptima ${ }^{\text {TM }}$ HPV Assay, Aptima ${ }^{\mathrm{TM}}$ HPV 16 18/45 and BD Onclarity ${ }^{\mathrm{TM}}$ HPV Assay).

\section{HYBRID CAPTURE}

The first method for the detection of HPV infection registered by the FDA (2003) is a type of solution hybridization followed by signal amplification, the Hybrid Capture II (HC2) generation method (Qiagen, Canada; former: Digene, USA) [8]. The materials to be examined are cervical swabs and biopsies [9]. The second-generation $\mathrm{HC}$ test allows to show the presence of 5 types of the virus with low oncogenic potential (HPV $-6,-11,-42,-43,-44)$ and 13 types of the virus with high oncogenic potential (HPV $-16,-18,-31,-33$, $-35,-39,-45,-51,-52,-56,-58,-59,-68)$. 


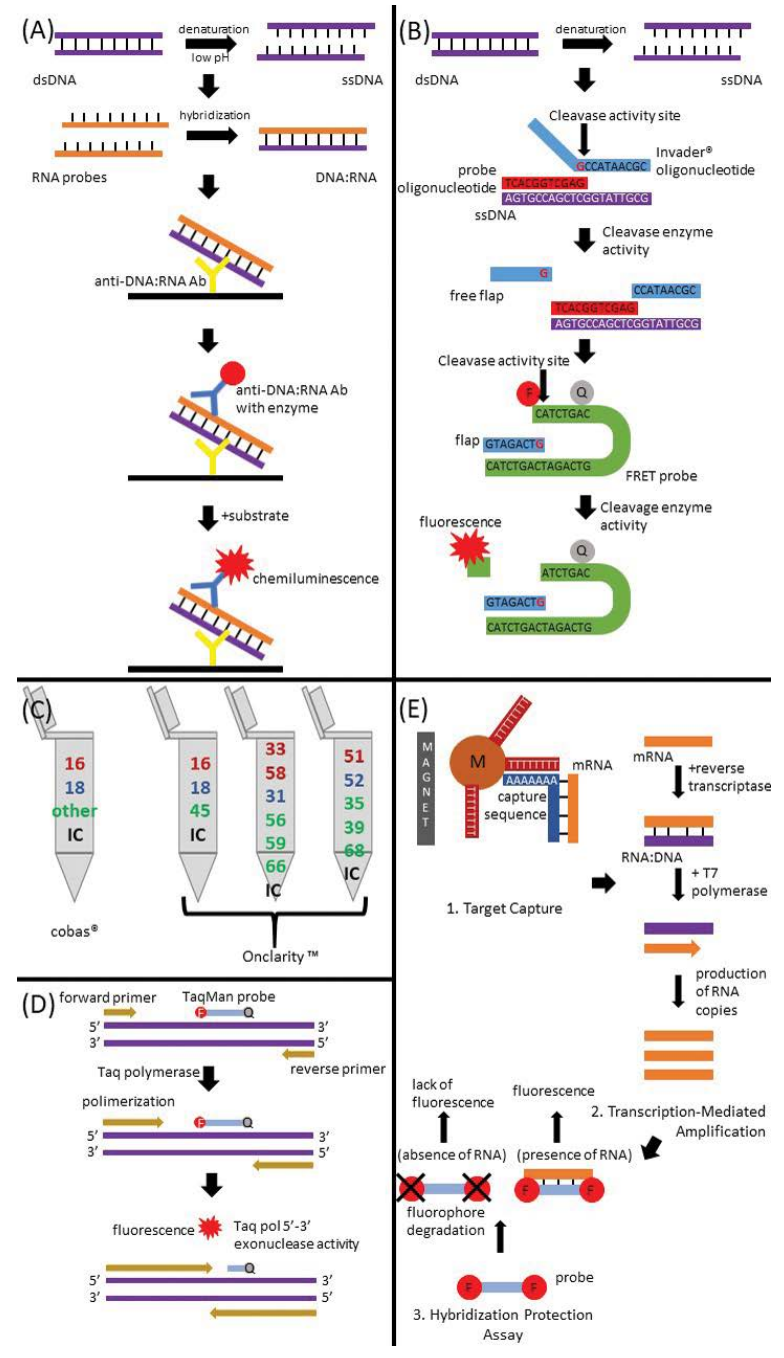

Figure 1. Principles of molecular HPV tests (description in the relevant paragraphs): (A) Hybrid Capture; (B) Cervista; (C) COBAS vs. Onclarity detection and genotyping of HPV types - different colors are different fluorescent dyes; (D) Common steps in COBAS and Onclarity; (E) APTIMA; F - fluorophore; Q - quencher; IC — internal control; $\mathrm{M}$ - magnetic microparticle

In the laboratory, the material is denatured in an alkaline environment [9] leading to lysis of cervical epithelial cells, viral capsid damage, release of HPV DNA and obtaining a single strand of viral DNA (Fig. 1A). The single strand of HPV DNA hybridizes with a specific RNA probe and, practically, with a mixture of RNA sequences complementary to HPV DNA of high or low oncogenic potential. The resulting RNA: DNA hybrids are captured and immobilized in wells of a microplate coated with antibodies against RNA:DNA hybrids. Then they are combined with a conjugate of anti-hybrid antibodies with alkaline phosphatase. The addition of a chemiluminescent substrate (dioxetane) to the enzyme reaction triggers the emission of light proportional to the number of hybrids. The luminous intensity is measured in the luminometer and expressed in relative light units (RLU) in relation to the emission of positive control light. In order to eliminate false negative results caused by too little material, the recommended cutoff value is $1.0 \mathrm{pg}$ of viral DNA per $1 \mathrm{~mL}$ of the test sample, this value is similar for each of the HPV types detected [9]. This concentration is equivalent to 5,000 viral copies per assay or 100,000 copies/mL [10].

The advantages of the test are: the semi-quantitative evaluation of viral DNA in the infected cell and a relatively high sensitivity of the method, comparable to the amplification reaction [11-13]. The method allows to distinguish virus types with high and low oncogenic potential but does not specify particular genotypes. The disadvantage of the test is also the occurrence of cross-reactions between the probe detecting HPV types with high oncogenic potential and other HPV viruses, the sequence of which do not contain a probe [14]. However, increasing the cutoff value to $10 \mathrm{pg}$ of viral DNA per $1 \mathrm{~mL}$ eliminates the majority of cross reactions, except for reactions with HPV -53 and -67 [14]. From a clinical point of view, cross-reactions of the probe detecting types with high oncogenic potential with non-oncogenic types have practically no effect on the treatment of patients with cytological changes in the cervix [15]. Another disadvantage of the test is the possibility of false negative results when using some antifungal creams and contraceptive jelly [9].

The literature also reports on the existence of the Hybrid Capture III test, which was intended to remedy the cross-reactivity problem by using labeled oligonucleotides instead of antibodies against the DNA:RNA hybrids used in the $\mathrm{HC} 2$ test [16]. Although it has come into commercial use, the small literature defines it as a "non-commercial test" and states that it is sometimes used in scientific research in combination with the $\mathrm{PCR}$ reaction and the $\mathrm{HC} 2$ test [17].

\section{CERVISTA}

Other methods approved by the FDA (2009) are the Cervista $^{\mathrm{TM}}$ HPV HR test and the Cervista ${ }^{\mathrm{TM}}$ HPV 16/18 test (Hologic Inc., USA). The materials for examination are cervical swabs [18], as well as biopsies [19].

The Cervista ${ }^{\mathrm{TM}}$ HPV HR test is based on solution hybridization and is a qualitative test to detect DNA of all 14 types of HR HPV $[18,19]$. The method uses the Cleavase enzyme and consists of two isothermal reactions: the primary one, i.e. the binding of oligonucleotides to the target sequence, and the secondary one, i.e. fluorescence generation [18]. In the primary reaction, two types of oligonucleotides are used: a probe oligonucleotide comprising a sequence complementary only to the 5 'part of the target sequence and a non-complementary region to its further part and Invader ${ }^{\circledast}$ oligonucleotide, complementary to the 3 ' part of the target sequence (Fig. 1B). These oligonucleotides overlap with at least one nucleotide, so that when bound to the target sequence, a structure is created that is a substrate for the Cleavase enzyme. This enzyme cleaves 
the non-complementary region and overlapping nucleotides from the oligonucleotide probe. In the secondary reaction, the cleaved fragment hybridizes to a FRET oligonucleotide with a hairpin structure. FRET oligonucleotide has a fluorophore and a quencher. The presence of the quencher eliminates the phenomenon of fluorescence, because its absorption spectrum coincides with the emission spectrum of the fluorophore [20].

The next sequence is created, which cleaves the Cleavase enzyme, because in this case, nucleotides of the hybridized sequences overlap. Cleavase cleaves the FRET oligonucleotide between the fluorophore and the quencher, which causes fluorescence emission [18]. The internal control of the test is the sequence encoding the histone 2 protein - the mixture of oligonucleotides also contains oligonucleotides that bind to this sequence. For the method to detect the presence of viral DNA and prevent false negative results, 1,250-2,500 copies of DNA are required for virus types $-16,-18,-31,-45,-52$ and $-56 ; 2,500-5,000$ copies of DNA for types $-33,-39,-51,-58,-59,-66$ and -68 ; and 5,000-7,500 copies for type -35 [18].

The Cervista ${ }^{\mathrm{TM}}$ HPV HR test is characterized by high analytical sensitivity, comparable to the sensitivity of the HC2 test [19]. Compared to the HC2 test, the advantages of the test are: the Cervista includes an internal control, requires lower sample volume and involves hands-free time, because there is a possibility for automation [21, 22]. Because the test requires a small-volume sample, the collected material can be used for a greater amount of analysis, e.g. for testing for other pathogens. The disadvantages of the test are: cross-reactivity with HPV types -67 and -70 and the possibility of false negative results when using contraceptive gels and antifungal creams. Like the HC2 method, Cervista is not specific for particular viral genotypes [18].

The Cervista ${ }^{\text {TM }}$ HPV 16/18 test is based on the same reactions as the Cervista ${ }^{\text {TM }}$ HPV HR test, however, it contains oligonucleotides complementary only to the two most oncogenic HPV strains: 16 and 18, so it is used to detect only these two types [23]. The test can be used alone or in combination with Cervista ${ }^{\mathrm{TM}}$ HPV HR, which is recommended in the case of squamous cells with indeterminate significance (ASC-US) [21]. The advantages of the test are high analytical sensitivity and analytical specificity [24]. In comparison to the PCR method, the overall positive and negative percentages of compliance were $94 \%$ and $85 \%$, respectively [24]. The disadvantage is the cross-reactivity with HPV 31; however, it only occurs at high concentrations of this genotype in the sample [21,22].

\section{COBAS}

The COBAS ${ }^{\circledR}$ HPV (Roche Molecular Systems Inc., Switzerland) was approved by the FDA in 2011. The material for examination is an LBC (liquid-based cytology) cervical swab [25]. The test contains primers that are used in the PCR reaction to amplify the sequence of about 200 nucleotides of the gene encoding the L1 protein of $14 \mathrm{HR}$ HPV types. Oligonucleotide primers are fluorescently labeled, allowing the use of quantitative PCR technology (qPCR). The reaction is automated and takes place in the dedicated COBAS $\times 480$ instrument, which reduces the manual work required [25]. There are 4 fluorescent probes used: separate for HPV-16, for HPV-18, for the remaining 12 types, and for the beta-globin gene as positive control of human DNA isolation (Fig. 1C). The test is therefore differentiating only for HPV -16 and -18 genotypes.

If L1 gene sequence of one or more HR HPV types is present in the sample, specific primers attach to the complementary sequences and the amplification reaction takes place (Fig. 1D). Detection is based on oligonucleotide probes [26]. These probes are labeled at one end with a fluorophore and at the other with a quencher. The quencher is so close to the fluorophore that no emission of fluorescence occurs. If the probe binds to a complementary sequence, then it will be degraded during the ongoing qPCR reaction, thanks to 5'-3' exonuclease activity of polymerase.

Degradation of the probe causes separation of the fluorophore from the quencher, thanks to which the fluorescence can be detected (for each marker at different excitation wave) [26].

The detection limit (LoD) has been specified for 150 copies $/ \mathrm{mL}$ for type $-45,300$ copies $/ \mathrm{mL}$ for types $-16,-31,-33$, $-39,-51$ and $-59,600$ copies/mL for types $-18,-35$ and -58 , 1200 copies/ $\mathrm{mL}$ for types $-56,-66$ and -68 and 2400 copies/mL for type -52 [25].

The advantage of the test is its high sensitivity, comparable to the HC2 test [27]. The COBAS test shows lower cross-reactivity with non-oncogenic virus types than Hybrid Capture II (1.2\% vs. 2.2\%) [28]. The test does not cross-react with other microorganisms or interact with lubricants or antifungal drugs [25]. The COBAS test allows genotyping of only HPV -16 and -18 types. The remaining 12 types give the same signal, so they are detected together, and it is not possible to differentiate the type of virus. Another advantage mentioned above is automation, which reduces the need for manual work. The literature does not report any shortcomings of the test, however, the high price of the instrument used to conduct the test can certainly be regarded as a disadvantage.

\section{APTIMA}

The APTIMA (Gen-Probe, USA) test was approved by the FDA in 2011. The materials tested are ThinPrep cervical smears [29]. The tests are designed to detect mRNA of E6/E7 oncoproteins encoded in the viral genome. There are two variants of this test: APTIMA ${ }^{\text {TM }}$ HPV and APTIMA ${ }^{\text {TM }}$ 
16 18/45 (approved by the FDA in 2012). APTIMA ${ }^{\text {TM }}$ HPV detects an infection with $14 \mathrm{HR}$ HPV types, while APTIMA ${ }^{\mathrm{TM}}$ 16 18/45 detects an infection with three HPV oncogenic types: $-16,-18$ and/or -45 . The tests do not allow to distinguish which of the detected types of infection occurred.

The APTIMA test consists of 3 stages, which are carried out in one tube: target capture; target amplification; detection of the amplification products [29]. At the beginning, samples are transferred to the Specimen Transport Medium, in which cell lysis occurs and the mRNA contained therein is released (Fig. 1E). Then, target mRNAs bind to complementary oligonucleotides with (poly-deoxyadenosine) polyA tail. Next, these hybrids are bound by poly-deoxythymidine (polyT) molecules, attached to the magnetic microparticles. This makes it possible to separate the target mRNA with a magnet. The next step, amplification, is associated with using the TMA method, i.e. amplification of RNA using reverse transcriptase and T7 polymerase. The captured mRNAs are transcribed into complementary DNA by reverse transcriptase. The cDNA contains a promoter for the T7 RNA polymerase, which allows this enzyme to join the CDNA and create multiple copies of the complementary RNA strand. Detection of the resulting amplicons is done using the Hybridization Protection Assay. The assay involves hybridization of duplicated sequences with fluorescently labeled oligonucleotide probes. In the absence of hybridization, the probe is degraded by borate buffered solution containing a surfactant. Therefore, the fluorescence signal can be detected only in the presence of multiplication by T7 polymerase. Light emitted by hybrids is measured by RLU using a luminometer [29].

The LoD test, according to the manufacturer's data, is less than 100 copies/reaction for types $-16,-18,-31,-33$, $-35,-39,-45,-58,-59,-66$, and -68 , and between 100 and 300 copies/reaction for types $-51,-52$ and -56 . The reaction is carried out in a volume of $400 \mu \mathrm{L}+/-100 \mu \mathrm{L}$ [29].

The sensitivity of the test is comparable to the sensitivity of the HC2 test (according to Ratman et al., 96.3\% for APTIMA vs $94.3 \%$ for Hybrid Capture II), so it is high, however, the greatest advantage of the test compared to $\mathrm{HC} 2$ is a higher correlation between a positive result of the test and pre-cancer/cervical cancer stages [30]. The test also has a higher specificity compared to the COBAS test [31]. A small disadvantage of the test is cross-reactivity with HPV types $-26,-67,-70$ and -82 , however, it does not show cross-reactivity with other HPV strains or microorganisms. Another disadvantage of the test is no genotyping of particular viral genotypes. The test interferes with some lubricants containing Polyquaternium 15, as well as with some antifungal agents containing tioconazole [29].

\section{ONCLARITY}

This test was approved by the FDA in 2018. The BD Onclarity ${ }^{\mathrm{TM}}$ HPV Assay (Becton, Dickinson and Company, USA) is based on qPCR [32]. The materials are cervical swabs collected in a BD SurePath Preservative Fluid. BD Onclarity ${ }^{\mathrm{TM}}$ HPV Assay detects E6/E7 oncogenes of 14 HPV HR types. The test is performed in three separate tubes (Fig. 1C). Onclarity test differentiates infection types $-16,-18,-31,-45,-51$ and -52 while the remaining 8 genotypes are detected as 3 different groups (-33/-58, -56/-59/-66 and 35/39/68) [32, 33]. The three tubes are necessary because the test uses 15 probes ( 14 for viral sequences and 1 for the human beta globin gene sequence, as internal control), but only four fluorescent dyes are employed so each tube contains different probes labeled with the markers used.

The test is fully automated and is divided into two stages. The first stage consists in cell lysis and DNA isolation in a high $\mathrm{pH}$ environment $[32,33]$. The second stage is based on TaqMan oligonucleotide probes, identically to the COBAS test (Fig. 1D).

The LoD of the test is about 250 copies/mL for HPV-16, in the range of 800-900 copies/mL for HPV $-31,-52$ and -66 , in the range of $1,000-1,500$ copies/mL for types $-18,-45$, -56 and -59 , in the range of $1,500-1,800$ copies $/ \mathrm{mL}$ for types $-33,-35,-39$ and -51 and in the range of 2,300-2,400 copies/ $\mathrm{mL}$ for types -58 and -68 [32].

The advantages of the test are high specificity and sensitivity, which are comparable to the HC2 test [34]. The test provides genotyping information for 6 types of HPV - this is the largest number out of all tests presented here. Thanks to full automation, the test is very easy to use and limits the work required [32]. There was also no cross-reactivity with other types of HPV or any microorganisms. As the only one of all FDA-accepted tests, it differentiates between 6 types of viral infection. The disadvantage of the test is the possibility of obtaining false negative results when using mucin, acyclovir and clindamycin [32]. Another downside is the high price of the BD Viper ${ }^{\mathrm{TM}}$ LT system, which is necessary to perform the test.

\section{SUMMARY}

All molecular tests approved by the FDA have high sensitivity and specificity. All tests detect 14 types of HPV HR, except for the HC2 test, which does not detect HPV-66, but does detect 5 low-oncogenic types. Cervista and APTIMA have variants that detect only types with the highest oncogenicity. The COBAS allows genotyping of HPV types -16 and -18 , while Onclarity allows genotyping of types $-16,-18,-31,-45,-51$ and -52 . The APTIMA test has the lowermost limit of detection among the tests described. The tests show cross-reactivity with low-risk HPV types, except for the Onclarity test, where cross-reactivity was not found. Cervista has the ability to be automated, and COBAS and Onclarity are compulsorily automated. Automation reduces the need to perform laboratory work, but the one-time expenditure for equipment is high. 
The main disadvantage of the described tests is that they do not allow for observation of mixed infections as well as monitoring of persistent infection with all types belonging to the HPV HR group.

In comparison to cytological and histopathological tests, the molecular HPV tests described show different correlations between pathological changes in the cervix and HPV-positive results. Differentiation of correlation between $\mathrm{CIN} 2+$ and positive HPV test result is as follows: HC2 test shows 93.4\% detection of CIN2+ lesions, Cevrista HPV HR 98.4\%, Cervista HPV 16/18 77\%, COBAS 95\%, APTIMA HPV $89.4 \%$, Onclarity $98 \%[10,21,27,30,33,35]$. The literature does not provide this data for the APTIMA 16 16/45 test.

Each of the tests described has both advantages and disadvantages. It is important, therefore, that a laboratory that wants to carry out HPV detection tests selects the most suitable option for itself.

\section{REFERENCES}

1. Bray F, Ferlay J, Soerjomataram I, et al. Global cancer statistics 2018: GLOBOCAN estimates of incidence and mortality worldwide for 36 cancers in 185 countries. CA Cancer J Clin. 2018; 68(6): 394-424, doi: 10.3322/caac.21492, indexed in Pubmed: 30207593.

2. Human Papillomavirus and Related Diseases Report. Barcelona: HPV Information Centre. http://www.hpvcentre.net/statistics/reports/POL. pdf (2018).

3. Bosch FX, Lorincz A, Muñoz N , et al. The causal relation between human papillomavirus and cervical cancer. J Clin Pathol. 2002; 55(4): 244-265, indexed in Pubmed: 11919208.

4. de Sanjosé S, Brotons M, Pavón MA. The natural history of human papillomavirus infection. Best Pract Res Clin Obstet Gynaecol. 2018; 47: 2-13, doi: 10.1016/j.bpobgyn.2017.08.015, indexed in Pubmed: 28964706.

5. Abreu ALP, Souza RP, Gimenes F, et al. A review of methods for detect human Papillomavirus infection. Virol J. 2012; 9: 262, doi: 10.1186/1743422X-9-262, indexed in Pubmed: 23131123.

6. Krajowa Izba Diagnostów Laboratoryjnych; Polskie Towarzystwo Ginekologiczne. Guidelines for application of molecular tests identyfying HR HPV DNA in the prevention of cervical cancer. Statement of experts from PGS (PTG) and NCLD (KIDL). Ginekol Pol. 2013; 84: 395-399, indexed in Pubmed: 23819408.

7. Olejniczak T, Rabiega-Gmyrek D, Niepsuj-Biniaś J, et al. [Human papilloma virus genotyping in women with abnormal cytology]. Ginekol Pol. 2015; 86(7): 541-546, indexed in Pubmed: 26376534.

8. Wong AA, Fuller J, Pabbaraju K, et al. Comparison of the hybrid capture 2 and cobas 4800 tests for detection of high-risk human papillomavirus in specimens collected in PreservCyt medium. J Clin Microbiol. 2012; 50(1): 25-29, doi: 10.1128/JCM.05400-11, indexed in Pubmed: 22090403.

9. Hybrid CaptureTM II. Gaithersburg: Digene Corporation. https://www. accessdata.fda.gov/cdrh_docs/pdf/P890064S009c.pdf. (2012).

10. Sargent A, Bailey A, Turner A, et al. Optimal threshold for a positive hybrid capture 2 test for detection of human papillomavirus: data from the ARTISTIC trial. J Clin Microbiol. 2010; 48(2): 554-558, doi: 10.1128/JCM.00896-09, indexed in Pubmed: 20007387.

11. Bozzetti MC, Nonnenmacher B, Mielzinska I, et al. Comparison between hybrid capture II and polymerase chain reaction results among women at low risk for cervical cancer. Annals of Epidemiology. 2000; 10(7): 466, doi: 10.1016/s1047-2797(00)00147-2.

12. Castle PE, Schiffman M, Wheeler CM. Hybrid capture 2 viral load and the 2-year cumulative risk of cervical intraepithelial neoplasia grade 3 or cancer. Am J Obstet Gynecol. 2004; 191(5): 1590-1597, doi: 10.1016/j. ajog.2004.05.018, indexed in Pubmed: 15547529.

13. Kulmala SM, Syrjänen S, Shabalova I, et al. Human papillomavirus testing with the hybrid capture 2 assay and PCR as screening tools. J Clin Microbiol. 2004; 42(6): 2470-2475, doi: 10.1128/JCM.42.6.2470-2475.2004, indexed in Pubmed: 15184422

14. Peyton CL, Schiffman M, Lörincz AT, et al. Comparison of PCR- and hybrid capture-based human papillomavirus detection systems using multiple cervical specimen collection strategies. J Clin Microbiol. 1998; 36(11): 3248-3254, indexed in Pubmed: 9774574.

15. Castle $\mathrm{PE}$, Solomon $\mathrm{D}$, Wheeler $\mathrm{CM}$, et al. Human papillomavirus genotype specificity of hybrid capture 2. J Clin Microbiol. 2008; 46(8): 2595-2604, doi: 10.1128/JCM.00824-08, indexed in Pubmed: 18579716.

16. Castle PE, Lorincz AT, Scott DR, et al. Comparison between prototype hybrid capture 3 and hybrid capture 2 human papillomavirus DNA assays for detection of high-grade cervical intraepithelial neoplasia and cancer. J Clin Microbiol. 2003;41(9): 4022-4030, indexed in Pubmed: 12958220.

17. Schiffman $M$, Glass AG, Wentzensen $N$, et al. A long-term prospective study of type-specific human papillomavirus infection and risk of cervical neoplasia among 20,000 women in the Portland Kaiser Cohort Study. Cancer Epidemiol Biomarkers Prev. 2011; 20(7): 1398-1409, doi: 10.1158/1055-9965.EPI-11-0206, indexed in Pubmed: 21602310.

18. Cervista ${ }^{\mathrm{T}}$ HPV HR. Madison: Third Wave Technologies. https://www. accessdata.fda.gov/cdrh docs/pdf8/p080014c.pdf (2008).

19. Kurian EM, Caporelli ML, Baker S, et al. Cervista HR and HPV 16/18 assays vs hybrid capture 2 assay: outcome comparison in women with negative cervical cytology. Am J Clin Pathol. 2011; 136(5): 808-816, doi: 10.1309/AJCPCQD7B7DPVHLH, indexed in Pubmed: 22031321.

20. Marras SAE. Selection of fluorophore and quencher pairs for fluorescent nucleic acid hybridization probes. Methods Mol Biol. 2006; 335: 3-16, doi: 10.1385/1-59745-069-3:3, indexed in Pubmed: 16785616.

21. Alameda F, Garrote L, Mojal S, et al. Cervista HPV HR test for cervical cancer screening: a comparative study in the Catalonian population. Arch Pathol Lab Med. 2015; 139(2): 241-244, doi: 10.5858/arpa.20140012-OA, indexed in Pubmed: 25611107.

22. Torres M, Fraile L, Echevarria Jm, et al. Human Papillomavirus (HPV) Genotyping: Automation and Application in Routine Laboratory Testing. Open Virol J. 2012; 6: 144-150, doi: 10.2174/1874357901206010144, indexed in Pubmed: 23248734.

23. Cervista ${ }^{\mathrm{T}} \mathrm{HPV}$ 16/18. Madison:Third Wave Technologies. https://www. accessdata.fda.gov/cdrh_docs/pdf8/P080015c.pdf (2008).

24. Bartholomew DA, Luff RD, Quigley NB, et al. Analytical performance of Cervista HPV 16/18 genotyping test for cervical cytology samples. J Clin Virol. 2011; 51(1): 38-43, doi: 10.1016/j.jcv.2011.01.016, indexed in Pubmed: 21376660.

25. cobas $^{\circledast}$ HPV Test. South Branchburg: Roche Molecular Systems. https:// www.accessdata.fda.gov/cdrh_docs/pdf10/p100020s017c.pdf (2015).

26. Rao A, Young $\mathrm{S}$, Erlich $\mathrm{H}$, et al. Development and characterization of the cobas human papillomavirus test. J Clin Microbiol. 2013; 51(5): 1478-1484, doi: 10.1128/JCM.03386-12, indexed in Pubmed: 23447640.

27. Heideman DAM, Hesselink AT, Berkhof J, et al. Clinical validation of the cobas 4800 HPV test for cervical screening purposes. J Clin Microbiol. 2011; 49(11): 3983-3985, doi: 10.1128/JCM.05552-11, indexed in Pubmed: 21880968.

28. Preisler S, Rebolj M, Ejegod DM, et al. Cross-reactivity profiles of hybrid capture II, cobas, and APTIMA human papillomavirus assays: split-sample study. BMC Cancer. 2016; 16: 510, doi: 10.1186/s12885-016-2518-4, indexed in Pubmed: 27439470.

29. APTIMA ${ }^{\oplus}$ HPV Assay. San Diego: Gen-Probe Incorporated. https://www accessdata.fda.gov/cdrh_docs/pdf10/P100042c.pdf (2011).

30. Ratnam S, Coutlee F, Fontaine D, et al. Aptima HPV E6/E7 mRNA test is as sensitive as Hybrid Capture 2 Assay but more specific at detecting cervical precancer and cancer. J Clin Microbiol. 2011; 49(2): 557-564, doi: 10.1128/JCM.02147-10, indexed in Pubmed: 21147950.

31. Castle PE, Eaton B, Reid J, et al. Comparison of human papillomavirus detection by Aptima HPV and cobas HPV tests in a population of women referred for colposcopy following detection of atypical squamous cells of undetermined significance by Pap cytology. J Clin Microbiol. 2015; 53(4): 1277-1281, doi: 10.1128/JCM.03558-14, indexed in Pubmed: 25653409.

32. BD Onclarity ${ }^{\mathrm{TM}}$ HPV Assay. Sparks: Becton, Dickinson and Company. https://www.accessdata.fda.gov/cdrh_docs/pdf16/P160037C.pdf (2018).

33. Ejegod DM, Junge J, Franzmann $M$, et al. Clinical and analytical performance of the BD Onclarity ${ }^{\mathrm{TM}} \mathrm{HPV}$ assay for detection of CIN2+ lesions on SurePath samples. Papillomavirus Res. 2016; 2: 31-37, doi: 10.1016/j. pvr.2016.01.003, indexed in Pubmed: 29074183.

34. Bottari F, Sideri M, Gulmini C, et al. Comparison of Onclarity Human Papillomavirus (HPV) Assay with Hybrid Capture II HPV DNA Assay for Detection of Cervical Intraepithelial Neoplasia Grade 2 and 3 Lesions. J Clin Microbiol. 2015; 53(7):2109-2114, doi: 10.1128/JCM.00246-15, indexed in Pubmed: 25903574.

35. Guo M, Khanna A, Feng J, et al. Analytical performance of cervista HPV 16/18 in SurePath pap specimens. Diagn Cytopathol. 2015; 43(4): 301-306, doi: 10.1002/dc.23221, indexed in Pubmed: 25352375. 